\title{
Thrombotic Microangiopathy Mediated by Complement in Postpartum Sequentially Treated With Plasmapheresis and Eculizumab
}

\author{
Orivaldo Alves Barbosa ${ }^{\mathrm{a}, \mathrm{e}}$, Andre Luis Coutinho de Araujo Macedo ${ }^{\mathrm{a}}$, Manoel Pedro Guedes Guimaraes ${ }^{\mathrm{a}}$, \\ Andre Costa Teixeira ${ }^{b}$, Raissa Marques Cavalcante ${ }^{\mathrm{c}}$, Priscila Dourado Evangelista ${ }^{\mathrm{a}}$, \\ Herivaldo Ferreira da Silva ${ }^{\text {, Jose Walter Correia }}{ }^{\mathrm{a}}$
}

\begin{abstract}
The complement-mediated thrombotic microangiopathy (TMA), also known as atypical hemolytic uremic syndrome (aHUS), is rare and potentially fatal, presenting as TMA and dysfunction/endothelial edema secondary to diffuse disruption of a fluid phase alternating complementary pathways. We report a case of a young woman with postpartum anuric renal failure, TMA and normal levels of ADAMTS13 with partial clinical improvement after plasmapheresis, in which treatment with eculizumab led to the complete reversal of the clinical manifestations. Our work includes a brief literature review on the diagnosis and management of TMA in puerperium.
\end{abstract}

Keywords: Thrombotic microangiopathy; Atypical hemolytic uremic syndrome; Postpartum; Eculizumab; Diseases

\section{Introduction}

Thrombotic microangiopathy (TMA) comprises a heterogeneous group of diseases that can be acquired or inherited, with different clinical manifestations and have to mark the microangiopathic hemolytic anemia with schizocytes, thrombocytopenia and organ dysfunction due to ischemia and/or endothelial dysfunction [1]. In the absence of intravascular devices, the microangiopathic hemolysis arteriole stenosis in microcirculation may occur for five main reasons: thrombosis von Willebrand multimers typically found in thrombotic thrombocytopenic purpura (TTP); obstruction of the circulation by platelet and fibrin thrombi found in disseminated intravascular coagulation (DIC); direct invasion of microcirculation by tu-

\footnotetext{
Manuscript accepted for publication June 19, 2015

anternal Medicine Service, Hospital Cesar Cals, Fortaleza, Ceara, Brazil

bArgos Pathology, Brazil

${ }^{\mathrm{c} A c a d e m i c}$ of Medicine, Hospital Cesar Cals, Fortaleza, Ceara, Brazil

${ }^{\mathrm{d}}$ Haemathology Service, Hospital Cesar Cals, Fortaleza, Ceara, Brazil

${ }^{\mathrm{e} C o r r e s p o n d i n g ~ A u t h o r: ~ O r i v a l d o ~ A l v e s ~ B a r b o s a, ~ I n t e r n a l ~ M e d i c i n e ~ S e r v i c e, ~}$

Hospital Cesar Cals, Fortaleza, Ceara, Brazil.

Email: orivaldo.alves.barbosa@gmail.com
}

doi: http://dx.doi.org/10.14740/jh210w mor cells; small vessel vasculitis, autoimmune or infectious etiology; endothelial dysfunction, which may be mediated by complement or Shiga-like toxin [1].

The period of pregnancy and postpartum eventually complicates these conditions, by immunological changes (predisposing the formation of autoantibodies), changes in clotting factors with decreased level of ADAMST13 enzyme, which cleaves factor multimers of von Willebrand, and own pregnancy (preeclampsia and clinical spectra). Most of the time, the differential diagnosis of these diseases is difficult, requiring a multidisciplinary approach for the best outcome of the patient.

We report a case of a young postpartum, with hypertensive disorders of pregnancy, with TMA initially conducted as TTP, with partial response to plasmapheresis and complete response to eculizumab.

\section{Case Report}

An 18-year-old female, previously healthy, term pregnant woman sought medical assistance with pressure peaks, lower limb edema and reports of supposedly generalized tonic-clonic seizures. She was treated with magnesium sulfate and performed by cesarean delivery, with newborn presenting good vitality. On the fifth day after surgery, the patient presented paleness, asthenia, dyspnea, altered level of consciousness, dysarthria, headache, nausea, vomiting, excessive sweating and anuria. The tests revealed anemia (Hb: $4.3 \mathrm{~g} / \mathrm{dL}$ ), thrombocytopenia $\left(35,000 / \mathrm{mm}^{3}\right)$ and elevated lactate dehydrogenase (LDH) $(9,667 \mathrm{U} / \mathrm{L})$.

She was then referred to Hospital Cesar Cals, where she was admitted to the intensive care unit (ICU) due to the worsening of symptoms. In the ICU, the hypothesis of TTP was raised, atypical hemolytic uremic syndrome (aHUS), antibody syndrome antiphospholipid catastrophic or elevated liver enzymes syndrome, thrombocytopenia and hemolysis (HELLP); the determination of ADAMTS13 (85\%) was requested and plasmapheresis was started.

After the fifth session of plasma exchange, her thrombocytopenia, anemia and elevated levels of LDH improved clinically, but she presented no significant improvement in renal function and remained oliguric and dependent on dialysis. After stabilization, the patient was referred to the ward for further diagnostic investigation. Renal ultrasonography revealed a mild 


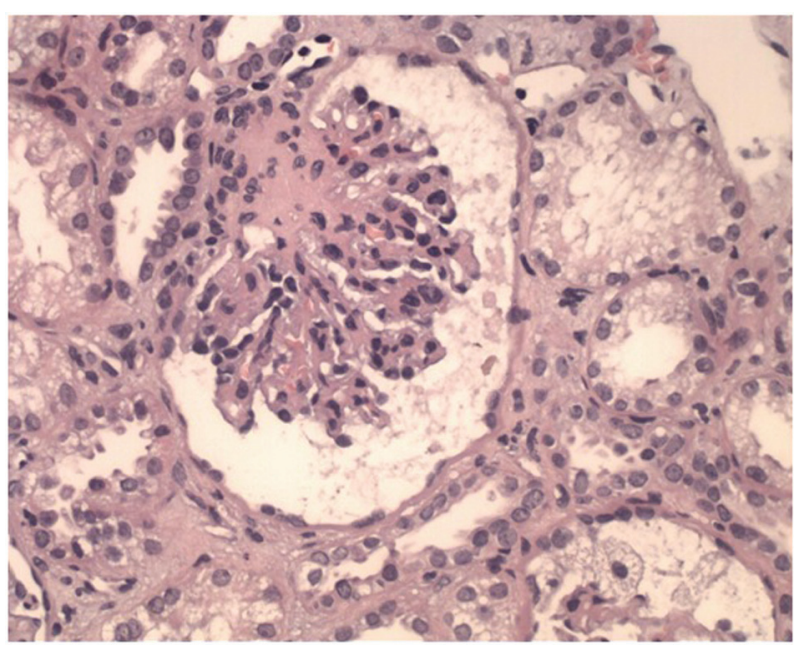

Figure 1. Glomerule with wrinkled loops associated with an ischemic process $(\mathrm{H} \& \mathrm{E}, \times \mathbf{4 0 0})$

increase of parenchymal echogenicity and the echocardiogram revealed systolic dysfunction with an ejection fraction of $42 \%$. Complement levels, antinuclear antibodies, serological tests for antiphospholipid antibodies and viral serology, such as hepatitis B and C, HIV and syphilis were negative.

With the diagnostic TMA at normal levels of ADAMTS13, predominant renal involvement was assessed as whether complement-mediated TMA indicated renal biopsy which evidenced slight interstitial fibrosis and tubular necrosis absence of intravascular thrombi (Fig. 1, 2), suggesting a diagnosis of aHUS, considering the lack of thrombosis in renal vasculature, as it reduces the possibility of TTP.

Eculizumab for the initiation of induction dose of 900 mg weekly for 4 weeks was determined. The patient's renal function and urine output improved, which increased to a level around 1,500 mL/24 h, after 4 days of the first dose of medication discontinued. She is now, 9 months after the initial episode, hemodialysis free and asymptomatic in regular infusion of eculizumab 1,200 mg every 2 weeks.

\section{Discussion}

Pregnancy and the postpartum period, by the physiological changes leading to decreased ADAMTS13 levels, supporting the emergence of TTP leading to immune deregulation, may form anti-FH antibodies, favoring the TMA-induced supplements, and anti-ADAMTS13. In the case of HELLP syndrome, pregnancy-specific, endothelial dysfunction occurs mediated by anti-angiogenic factors such as FMS-like tyrosine kinase, leading to microangiopathy syndrome unresponsive to the varying severe plasmapheresis.

The complement-mediated TMA, also known as aHUS, was first described in 1975 as a TMA with severe renal impairment and poor prognosis in patients with HUS and Shiga-toxin (STEC) negative [2]. We now know that this group of patients have a defect in the regulation of the activation of the fluid phase of the alternate complementary pathway, leading to the

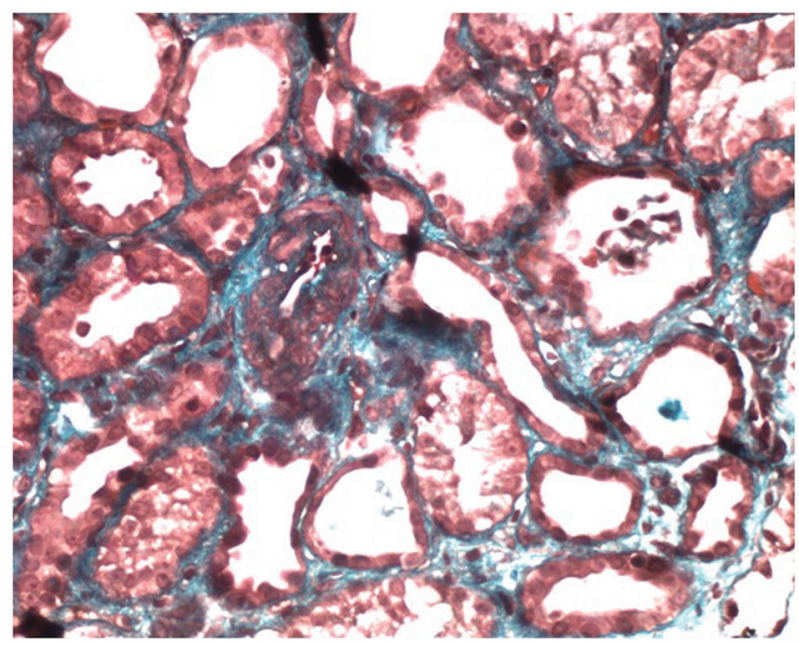

Figure 2. Arteriole with media thickening, endothelial edema and partial occlusion of lumen, features associated with microangiopathy (Masson Trichrome, $\times 400$ ).

activation of $\mathrm{C} 3 \mathrm{~b}$ and to the deposit the same tissue, causing activation of $\mathrm{C} 5$ and formation of the membrane attack complex (MAC) with secondary endothelial dysfunction. Most of these patients $(90 \%)$ have a genetic defect, the main deficiency of factor $\mathrm{H}(\mathrm{FH})$ and more rarely factor I, factor B and CD46, all regulators inactivation of $\mathrm{C} 3$. In $10 \%$ of cases, the defect is acquired, as anti-factor antibodies $\mathrm{H}$. These most commonly begin the picture in childhood patients, but in some cases, as described, can begin in adulthood after a trigger, such as infection, pregnancy and surgical stress.

By having a higher prevalence and being considered an emergency, TTP should always be considered for treatment with plasma exchange increases survival of $10-15 \%$ to $90 \%$. In cases of TMA by supplement, it may be partial response to plasmapheresis (around 50\%) for spare factor $\mathrm{H}$ autoantibodies and withdrawal [2]. In our case, the important renal involvement with anuria, the normal levels of ADAMTS13 and the discrete response to initial treatment for TTP led to the indication of renal biopsy, which showed microangiopathy without renal arteriolar thrombi, suggesting TMA by complement. The analysis of mutations and antibodies against the regulatory factors may be requested, but there are several mutations described and the negativity of this research does not exclude the diagnosis [1].

Eculizumab is an anti-C5 monoclonal antibody that prevents the formation of MAC, and the studies based on such cases showed decreased progression of renal disease [1-6]. In our case, the response was extremely positive because, on the fourth day after the infusion, the patient recovered diuresis and does not need more renal replacement therapy.

\section{Conclusion}

Our case emphasizes the importance of investigating extensively the TMA in the postpartum period, since the conduction in each patient differs and they are serious diseases that affect young people of childbearing age. 
Despite the high cost of medication prescribed to the patient, the benefit, both in functionality and in potential years of life gains outweighs the risk and the cost of prolonged hemodialysis and kidney transplantation.

\section{References}

1. George JN, Nester CM. Syndromes of thrombotic microangiopathy. N Engl J Med. 2014;371(7):654-666.

2. Taylor CM, Machin S, Wigmore SJ, Goodship TH. Clinical practice guidelines for the management of atypical haemolytic uraemic syndrome in the United Kingdom. $\mathrm{Br}$ J Haematol. 2010;148(1):37-47.
3. Waters AM, Licht C. aHUS caused by complement dysregulation: new therapies on the horizon. Pediatr Nephrol. 2011;26(1):41-57.

4. Nurnberger J, Philipp T, Witzke O, Opazo Saez A, Vester U, Baba HA, Kribben A, et al. Eculizumab for atypical hemolytic-uremic syndrome. N Engl J Med. 2009;360(5):542-544.

5. Park SJ, Shin JI. Beneficial effect of eculizumab on thrombotic microangiopathies: another point of view. Am J Kidney Dis. 2014;63(1):166-167.

6. Schmidtko J, Peine S, El-Housseini Y, Pascual M, Meier P. Treatment of atypical hemolytic uremic syndrome and thrombotic microangiopathies: a focus on eculizumab. Am J Kidney Dis. 2013;61(2):289-299. 